\title{
ENSEIGNEMENT DE LA COMBUSTION DANS L'OPTIQUE DU DÉVELOPPEMENT DURABLE
}

\author{
Bruno Detuncq \\ Professeur adjoint département de génie mécanique, École Polytechnique de Montréal \\ bruno.detuncq@polymtl.ca
}

\begin{abstract}
Résumé
L'enseignement de la combustion ne peut plus se faire maintenant comme elle se faisant autrefois. La prise en compte de la diminution des réserves et des conséquences des émissions doivent être traités comme un tout dans un cours de premier cycle en génie portant sur la combustion. Un questionnement sur les enjeux sociaux économiques et environnemental doit être intégré au cours de façon à dégager une vision globale du phénomène. Tendre vers un équilibre des différentes parties est un objectif important.
\end{abstract}

Mots clef: Limite des ressources non renouvelables, impact des émissions, concept de cours, intégration de connaissance, combustion et valeurs.

\section{INTRODUCTION}

Voici une déclaration qui a été faite par le ministre fédéral de l'énergie, monsieur Joe Oliver, le 12 avril 2013 : « La demande mondiale de pétrole joue en faveur des sables bitumineux et les craintes au sujet du réchauffement climatique commencent à s'estomper » [1]. L'ensemble des propos qu'a tenus le ministre Oliver à cette occasion indique que celui-ci ne voit pas de limite au marché du pétrole bitumineux et il s'est appuyé sur les analyses de l'Agence internationale de l'énergie [2] pour faire un portrait radieux de l'avenir de l'industrie la plus controversée au pays.

Selon le dernier rapport de l'Agence internationale de l'énergie, la demande mondiale devrait augmenter de 36\% d'ici 2035, principalement à cause des pays émergents. Cependant, cette même agence affirme aussi qu'il faut laisser dans le sol $70 \%$ des réserves d'hydrocarbures si on veut stabiliser l'augmentation de la température à moins de $2^{\circ}$ Celsius d'ici 2050 et ainsi éviter des « changements

CEEA13; Paper 001

Montréal, Qc; June 17-20, 2013 climatiques dangereux », selon les termes de l'Accord de Copenhague sur le climat. Autres chiffres très significatifs, en 1950, il y avait environ 70 millions de véhicules en circulation dans le monde. En 1980, on en était à 410 millions et en 2011 le chiffre atteignait presque 1 milliard.

On constate dans ces données et dans ces affirmations du ministre Oliver une contradiction fondamentale liée au mode de vie moderne. La contradiction est entre notre aspiration au bien-être et la protection des ressources et de l'environnement. La combustion est au cœur de ce mode de vie et au cœur de cette contradiction. Cet outil de progrès est en train de devenir le tombeau de l'avenir. La civilisation qui a été bâtie depuis 300 ans est adaptée à un climat donné et à un environnement abondant, les humains sont en train de modifier l'équilibre planétaire de façon importante, la civilisation de l'avenir pourra-t-elle s'adapter à des conditions qui risquent d'être très différentes ?

\section{CONCEPT DU COURS}

Le phénomène de la combustion fait intrinsèquement partie de notre monde industriel; 85 \% de toute l'énergie primaire utilisée par les humains provient des combustibles fossiles. Cependant, depuis 30 ans, il n'est plus possible de dissocier le phénomène énergétique des conséquences environnementales. Dans un tel contexte, comment enseigner la combustion à de futurs ingénieurs et comment intégrer les préoccupations concernant le développement viable?

Force est de constater que tous les manuels traitant de la combustion que j'ai consultés et s'adressant à des étudiants de génie le font de façon presque exclusivement centrée autour des notions de réactions chimiques, d'équilibres chimiques, de vitesse de flamme et de quelques applications portant sur les flammes, les brûleurs ou les moteurs. 
Très peu de ces livres abordent les problématiques de réserves des combustibles fossiles et de pollution émise par la combustion. Ce qui se passe en amont du phénomène thermochimique et ce qui se passe à la sortie du lieu d'utilisation n'a plus d'intérêt. Ces phénomènes sont relégués à d'autres cours spécialisés, il n’y a donc plus de vision globale de la chose. C'est à mon avis une erreur importante, car la majorité des ingénieurs, qu'importe leur champ de travail, seront plus souvent confrontés à des questions relatives au choix de filières énergétiques et de réglementation concernant les émissions qu'à des problématiques de design de composantes spécifiques d'une installation.

Plusieurs très bons livres portant sur la combustion ont été publiés depuis la fin des années 90 , les auteurs sont des spécialistes des phénomènes fondamentaux et poursuivent des travaux de recherche dans ces domaines; leurs livres reflètent cette approche et sont donc destinés, à mon avis, à un public étudiant de $2^{\mathrm{e}}$ et $3^{\mathrm{e}}$ cycle se destinant à la recherche ou à l'application dans un domaine pointu. Est-ce l'approche nécessaire pour des étudiants finissant un premier cycle universitaire ? Ma réponse est non. Il est nécessaire d'aborder l'ensemble de la problématique de la combustion ainsi que les débats sociaux qui l'entourent pour que les jeunes ingénieurs puissent être sensibilisés aux tenants et aux aboutissants de la combustion et à la réalité complexe des filières énergétiques reliées aux combustibles fossiles. Si cela est fait, il y a alors place à des cours gradués portant sur les éléments spécifiques des flammes turbulentes et des brûleurs pour ceux qui se destinent à l'étude et au design des systèmes de combustion modernes.

Un cours de premier cycle portant sur la combustion peut et doit être un lieu où un certain débat de société se fait. Ce ne peut pas être un cours de spécialisation avancé, restreint uniquement à la part technicoscientifique d'extraction du maximum d'énergie. Même si l'aspect de l'efficacité énergétique lors de la combustion est très important, il ne doit pas prendre toute la place au détriment des aspects environnementaux, sociaux et politiques.

Afin de dispenser une formation couvrant un spectre plus large de la problématique de la combustion, j'ai développé un cours où j'essaie de trouver un équilibre entre ces différents objectifs. Dans la première partie j'aborde les sujets suivants :
- La définition et le classement des différents types d'énergie

- L'historique de la consommation par filière énergétique

- Les projections dans l'avenir des réserves restantes et des tendances futures

- Les conséquences des choix qui peuvent être faits.

À titre d'exemple, étant donné le développement des sables bitumineux de l'Alberta, le gouvernement du Québec doit se demander s'il faut importer ce pétrole et favoriser cette filière, ou faire d'autres choix ? La réalité nous oblige à faire face simultanément aux enjeux économiques, politiques et écologiques se font face et il n’y a pas de réponse simple. Aborder cette problématique en classe permet au moins d'exposer la complexité des enjeux et d'ouvrir un débat sur les valeurs.

Dans la deuxième partie du cours, j'aborde la partie classique de la combustion proprement dite, mais en limitant quelque peu cette matière pour conserver du temps pour la dernière section portant sur les effets de la combustion. Les aspects couverts sont les suivants :

- La combustion stoechiométrique et nonstoechiométrique

- Le calcul de l'équilibre chimique par la méthode des constantes d'équilibre et par la méthode de la minimisation de la fonction de Gibbs par les multiplicateurs de Lagrange

- $\quad$ La vitesse de réaction chimique

- $\quad$ La vitesse de flamme laminaire

- Quelques applications portant sur les jets, les flammes et les brûleurs

- $\quad$ Une introduction sur les fours industriels

La troisième partie du cours porte sur la pollution et ses effets. Dans cette section, est couvert les sujets suivants :

- L'effet de serre et les différents gaz qui participent à cet effet

- La relation de l'effet de serre avec la consommation des combustibles fossiles

- Les pluies acides et le phénomène de smog, comment sont générés ces phénomènes et comment on peut les diminuer

- Les particules émises par la combustion

- Les autres gaz, $\mathrm{CO}, \mathrm{SO}_{2}, \mathrm{NOx}$, les effets sur la santé de ces différents corps, leur mode de production et les méthodes de séquestration. 


\section{CAPSULES D'INFORMATION}

La matière couverte par le cours se prête facilement à un questionnement sur les enjeux sociaux de la combustion et de ses conséquences. Lorsque j’ai mis en place le cours en 1995, très peu d'articles de journaux portaient sur la relation entre combustion et pollution. C'est maintenant très différent. Je procède à une actualisation de l'information chaque semaine à l'aide d'une revue de presse portant sur un des thèmes couverts dans le cours. De nombreux articles paraissent chaque semaine et chaque mois dans des journaux et dans des revues grand publique. Je fais également une revue de livres portant sur les aspects sociaux liés aux modifications climatiques et à la pollution atmosphérique pour montrer que de nombreux groupes de recherches alimentent la discussion à ce niveau.

Régulièrement au début du cours, je place une capsule d'information de quelques minutes sur les actualités dans le domaine des énergies ou de l'environnement ou sur un livre ouvrant un thème de réflexion. C'est d'ailleurs par ce procédé que j'ai amorcé la présentation d'aujourd'hui. La participation des étudiants est également sollicitée en termes d'expérience personnelle ou d'information à transmettre à l'ensemble de la classe.

Je fais également appel à des études qui abordent des thèmes plus larges qui portent par exemple sur les tendances sociologiques importantes comme l'accroissement de la population mondiale ou le nombre d'enfants par famille à travers le monde, et l'impact sur les tendances à moyen et à long terme sur la consommation.

En support au cours, un site web est conçu de façon à être accessible à tous, étudiants de Poly ou personnes de l'extérieur. J'y accumule des informations classées par thèmes et j’y ai placé également des hyperliens donnant accès à de nombreux autres sites portant sur les différents aspects du cours. Ce site de cours permet aux étudiants de vérifier les sources que j'utilise et d'enrichir les connaissances de ceux qui désirent pousser plus loin leurs recherches d'informations et leur étude personnelle pour leurs besoins personnels ou professionnels. Une autre partie du site n'est accessible qu'aux étudiants du cours.

\section{CONCLUSION}

La résistance au changement est normale si ce changement est perçu comme une perte. La combustion est synonyme de confort, de bien-être, de plaisir de consommer et de facilité de déplacement. Il n'est pas facile de mettre de côté volontairement ces choses. Il ne faut cependant pas attendre que la nature nous y force. Les ressources sont limitées, les combustibles fossiles sont épuisables, on passe de réserves faciles à extraire à des réserves de plus en plus difficiles, coûteuses et polluantes à exploiter. Par ailleurs, la pollution et l'augmentation de l'effet de serre seraient très longues à se résorber si on arrêtait maintenant d'émettre les gaz polluants. Si on entreprend une lente diminution des émissions, ce sera encore plus long. Comment se soucier des générations à venir ? Il est très difficile de se sentir responsable des effets de nos gestes si les conséquences seront senties 5, 10, 20 ou 50 ans plus tard.

Entendre parler du réchauffement du climat et de la diminution des ressources peut, à la longue, diminuer l'intérêt que le public porte à ces sujets. La répétition de l'information sur les dérèglements climatiques sans lien clair avec le quotidien a pour effet de réduire la sensibilité des citoyens à ces phénomènes qui ont et auront véritablement des conséquences sur eux. Même l'augmentation régulière du prix de l'essence, on s'en accommode. Les gens ne veulent pas entendre tout le temps parler de malheurs, et c'est normal. Mais comment faire passer le message, et à qui ?

Il est impératif que les ingénieurs formés actuellement aient cette sensibilité, et qu'elle soit encrée dans une connaissance scientifique qui donne une assise solide à la réflexion et une capacité de garder espoir. Ces connaissances leur permettront de suivre l'évolution des débats concernant les enjeux planétaires, car ce sont eux qui prendront les décisions importantes dans les années à venir concernant l'énergie durant cette étape historique qui risque d'être très difficile à ce sujet.

Notre monde est dépendant des combustibles fossiles et cela entraîne des choix difficiles. Est-il possible de se débarrasser rapidement de cette source d'énergie ? Non, mais il faut, en connaissant les tenants et aboutissants de cette filière, travailler pour que chaque développement tende à diminuer sa présence à moyen terme. Ce peut être par des taxes sur les combustibles et sur les produits utilisant ces combustibles, dont les revenus seront utilisés pour promouvoir la conservation et le développement d'autres sources énergétiques, ou d'autres modes d'action, mais il est nécessaire que ceux qui prendront les décisions à l'avenir aient une vision globale des enjeux.
CEEA13; Paper 001

Montréal, Qc; June 17-20, $2013 \quad$ - 3 of 4 - 
Le cours de « Combustion et pollution atmosphérique » est offert une fois par année aux étudiants de génie mécanique en $4^{\mathrm{e}}$ année dans les orientations Énergie et Mécanique du Bâtiment, ainsi qu'aux étudiants du programme de DESS en Développement Durable, option Énergie. Également, plusieurs étudiants étrangers inscrits à Polytechnique en échange suivent le cours. Le nombre d'étudiants est en moyenne de 50, ce qui est appréciable pour un cours optionnel. J'en déduis donc que ce cours est utile et est perçu comme utile par les étudiants. Cette impression est corroborée par les évaluations du cours faites par les étudiants qui apprécient l’aspect ‘ouverture’.

\section{Remerciements}

Je tiens à remercier chaleureusement madame Louise Leduc pour son aide et ses conseils très pertinents lors de la rédaction du texte.

\section{Références}

[1] Journal La Presse ; 12 avril 2013

[2] World Energy Outlook; 2013

CEEA13; Paper 001

Montréal, Qc; June 17-20, 2013 - 4 of 4 - 